\title{
Enhanced Nonlinear Dynamics for Accurate Identification of Stiffness Loss in a Thermo-Shielding Panel
}

\author{
Bogdan I. Epureanu* and Shih-Hsun Yin ${ }^{\dagger}$ \\ Department of Mechanical Engineering \\ University of Michigan \\ 2350 Hayward Street, Ann Arbor, MI 48109-2125
}

\begin{abstract}
The dynamics of a panel forced by transverse loads and undergoing limit cycle oscillations and chaos is investigated. The nonlinear von Karman plate theory is used to obtain a model for healthy and damaged panels. Damage is modeled by a loss of stiffness in a portion of the plate. The presence of low levels of damage is identified by using an external nonlinear excitation and analyzing the attractor of the resulting dynamics in state space. Most of the current studies of such problems are based on linear theories and linear structures. In contrast, the results presented are obtained by using and enhancing nonlinear and chaotic dynamics, and have the advantage of an increased accuracy in detecting damage and monitoring structural health.
\end{abstract}

\section{Nomenclature}

panel width

external damping (dimensional)

external damping (nondimensional)

internal damping (dimensional)

internal damping (nondimensional)

coefficient characterizing the bending

stiffness of the panel (healthy)

coefficient characterizing the bending

stiffness of the panel (damaged)

Young's modulus

transversal force (nondimensional)

panel thickness

panel length

axial in-plane tension load

distributed transversal force (dimensional)

axial in-plane pre-load (nondimensional)

coefficient characterizing stretching induced

by bending

stiffness reduction factor

time (dimensional)

axial displacement (dimensional)

transversal velocity (nondimensional)

transversal displacement (nondimensional)

transversal displacement (dimensional)

axial coordinate (nondimensional)

axial coordinate (dimensional)

transversal coordinate (nondimensional)

transversal coordinate (dimensional)

nonlinear factor in the feedback excitation

linear factor in the feedback excitation

$$
\begin{aligned}
& \nu \\
& \rho \\
& \tau \\
& \sigma_{x x} \\
& \epsilon_{x x} \\
& \zeta
\end{aligned}
$$

\author{
Poisson's ratio \\ panel material density \\ time (nondimensional) \\ stress in the plate ( $x x$ component) \\ strain in the plate ( $x x$ component) \\ panel material damping constant
}

\section{Introduction}

The identification of stiffness loss in a plate is needed in several applications of structural health monitoring. In such applications, the use of non-destructive evaluation is exploited for detecting structural changes which indicate damage. ${ }^{1,2}$ In general, the ability to quantify and measure structural integrity is an important advantage as it increases safety while reducing operation costs and lengthening the lifetime of aging assets.

Identifying loss of stiffness is one of the most common means to detect damage. In turn, detecting the loss of stiffness is accomplished by monitoring a feature of the system. Vibration responses (frequency response functions, mode shapes and natural frequencies), electromagnetic properties, ultrasound, are examples of such features. The most common monitoring techniques are focused on detecting the presence of damage without any concern for its precise location, level or extent. In such approaches, the feature for a healthy and a damaged system are compared, and the identified differences usually are not quantified, but they are used as a red/green indicator. More advanced techniques use location-dependent features and focus on the identification of the location and/or the extent of damage. Such techniques require distributed sensors, more comprehensive information about the system, and a quantifiable feature whose measure may

${ }^{*}$ Member AIAA, Email: epureanu@umich.edu

†Email: syin@umich.edu 


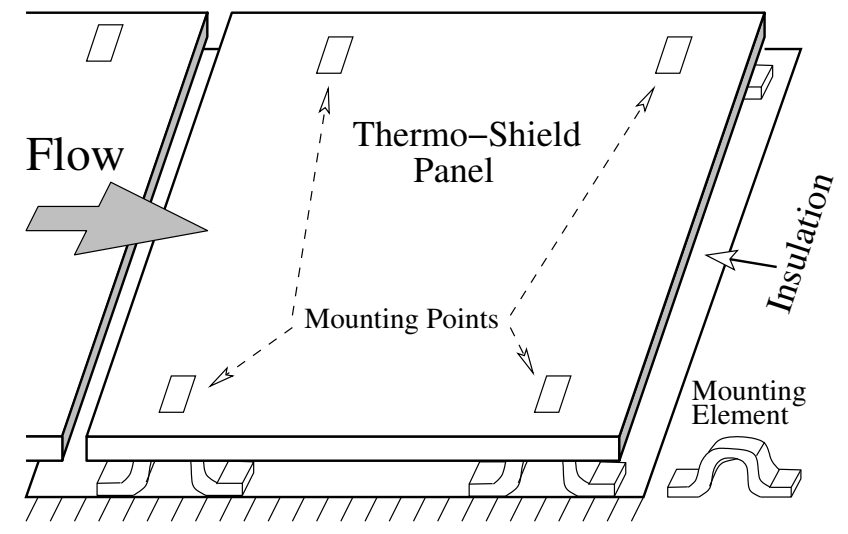

Fig. 1 Thermo-shielding panel subjected to internal thermal loads and interacting with an external (supersonic) flow (i.e. an aeroelastic system)

be correlated with the extent and location of damage. Nevertheless, such techniques are the most advantageous because they allow for the estimation of the remaining life of the system, and are the basis for the elimination of time-scheduled inspections, and a transition from time-based to condition-based maintenance.

Monitoring structural integrity is particularly important in aerospace applications ${ }^{3-5}$ where detecting the onset and propagation/evolution of damage during normal operation of the monitored system is highly desired (e.g. monitoring air-frames, unmanned air vehicles, the joint strike fighter, buildings and bridges). Among the most important technical difficulties faced by current methods are detecting incipient damage (of small level) and taking adequate measurements during normal operating conditions. These issues have limited the available techniques to mostly offline detection of large damage.

Most current techniques for damage detection are vibration-based, i.e. they are focused on determining changes in the frequencies and modes of vibration. ${ }^{1,3,6-8}$ Other similar techniques use wavelet analyses, ${ }^{9-12}$ Ritz vectors, ${ }^{2,13}$ stochastic approaches, ${ }^{14,15}$ subspace updating, ${ }^{16-23}$ or evolutionary algorithms. ${ }^{24}$ All these methods have been developed for monitoring linear structures. Much fewer methods are currently available for detecting damage in nonlinear structures. Most of the nonlinear methods are based on system identification, ${ }^{25-31}$ while a few use neural networks, ${ }^{32-34}$ or Lyapunov exponents. ${ }^{35-38}$ These nonlinear methods have important limitations, e.g. some have difficulty tackling high-dimensional systems, others do not predict the damage location or level, or do not detect simultaneous damages. However, our results show that the use of nonlinearities holds a great potential. We have observed that the changes in the shape of attractors of the dynamics are large for nonlinear systems and may be used for damage detection.

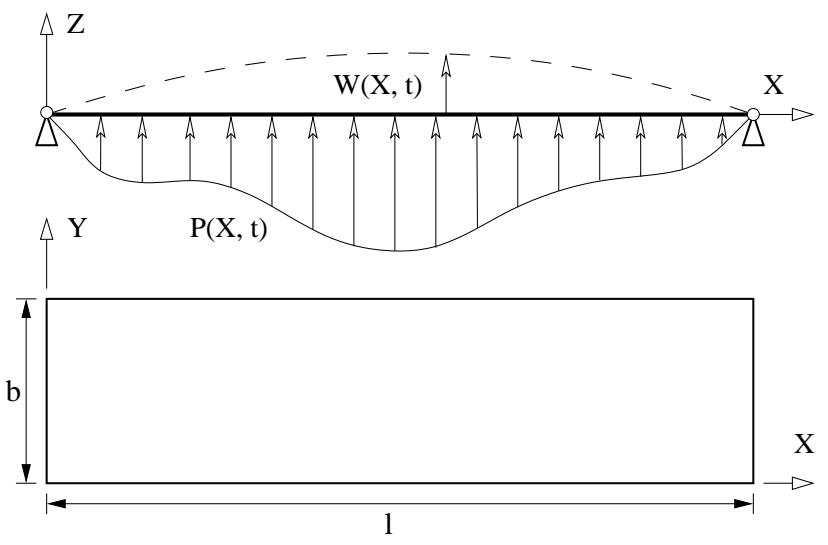

Fig. 2 Two-dimensional panel subject to transverse loading

For example, results have been obtained for a pinnedpinned panel with structural nonlinearity depicted in Fig. 1 and Fig. 2. These results are shown in Fig. 3 and Fig. 4 to demonstrate that the nonlinear dynamics of the panel excited harmonically changes dramatically when the panel stiffness changes. A mere $4 \%$ change leads to qualitative (large) changes in the attractor shape as shown in Fig. 3. Moreover, the attractors change with both the location and extent of damage. Loss of stiffness in a portion of the plate is simulated. A small loss of stiffness (10\%) along a small extent (5\% of panel length) can clearly be distinguished in Fig. 4 from larger damage levels (50\% loss) and larger damage extents (20\% length). In contrast, linear methods cannot detect the damage and also model incorrectly the panel (e.g. they predict inaccurate vibrations or conclude that the plate breaks, as shown for example in Fig. 4 where differences between the limit cycle oscillations of the nonlinear healthy panel and the linear vibrations are observed).

The main reasons for the increased need for nonlinear vibration-based damage detection are: $(i)$ nonlinearities are a pervasive presence in many systems affected by damage, (ii) linear techniques are not accurate when applied to nonlinear systems because often the presence or level of damage cannot be distinguished (by linear methods) from the nonlinear healthy system dynamics, (iii) current damage detection approaches do not take advantage of nonlinear dynamics, but minimize its influence, although nonlinear-based methods can provide greater sensitivity and performance.

Nonlinear phenomena are important not only in large but also in small amplitude vibrations when the strength of nonlinearity is high. An example of such a nonlinearity often found is a crack in a plate or a loose joint which may lead to changes in linear characteristics such as mode shapes, ${ }^{39}$ stiffness and damping, and can also induce nonlinear dynamics by introducing Coulomb friction and allowing the opening and closing 


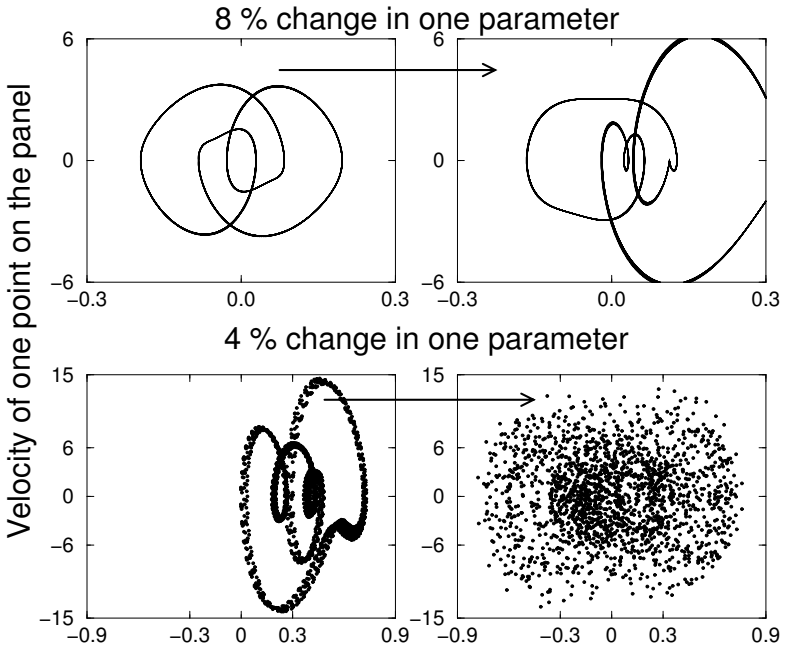

Displacement of one point on the panel / panel thickness

Fig. 3 Nonlinear systems are sensitive to parametric variations, and small parameter variations lead to dramatic changes in attractor shapes

of a gap (piece-wise linear dynamics with possible impacts $\left.{ }^{40-42}\right)$. Also, linear methods have difficulties because they are independent of the amplitude of vibration, and thus, the stiffening or softening of a healthy system due to nonlinearities may be erroneously assumed to be caused by damage. Furthermore, the co-existence of multiple attractors (for the same undamaged structure under the same excitation and) for the dynamics of a point on a panel with structural nonlinearity excited by unsteady flow, ${ }^{43}$ may be erroneously interpreted by linear methods as being due to damage. Additionally, nonlinear systems are more sensitive to parametric changes than linear systems (especially in the neighborhood of bifurcations ${ }^{44-46}$ ). Also, nonlinear and chaotic vibrations have been observed in industries such as aerospace, nuclear, telecommunications and sensors (wings, ${ }^{47-51}$ panels forced by flow-induced loads,${ }^{43,}{ }^{52-58}$ rotors,${ }^{59}$ pipes,${ }^{60-62}$ heat exchangers, ${ }^{60,63-65}$ micro-systems ${ }^{66,67}$ such as microplates), and aerospace technology (thermo-shielding panels $\left.{ }^{43}\right)$.

\section{Modeling}

A thermo-shielding panel mounted on a rigid substructure and subjected to unsteady aerodynamic forces is depicted in Fig. 1. A simplified model for the panel is shown in Fig. 2. The deformation of the panel occurs primarily in the transverse direction $(Z$ axis) and that deformation is considered of the order of magnitude of the panel thickness. Although this deformation is small, structural nonlinearity is present (and it is strong) due to the coupling between stretching and bending of the panel. Energy dissipation of the aeroelastic system is also considered in the model by accounting for internal and external damping. Finally, the damaged regions of the panel are modeled as exhibiting a local reduction in the bending stiffness of the panel.

\section{Structural Model}

The panel shown in Fig. 2 is modeled as a one dimensional, homogeneous, isotropic, and elastic thin plate with pinned-pinned end points. The thickness $h$ is considered much smaller than the length $l$. Also, the width $b$ is considered much larger than the length $l$. These assumptions hold for many cases of practical interest (such as a thermo-shielding panel). Nevertheless, the proposed approach for the accurate identification of loss of stiffness may be applied equally well to other cases, where these assumptions do not hold. For a linear elastic isotropic material, the stress-strain relation may be simplified (based on the assumption above) and expressed as

$$
\sigma_{x x}=\frac{E}{l-\nu^{2}} \epsilon_{x x}
$$

where $E$ is Young's modulus and $\nu$ is Poisson's ratio. To model the structural nonlinearity caused by the in-plane stretching of the panel due to bending, the von Karman's strain-displacement relation is used as follows

$$
\epsilon_{x x}=\frac{\partial U}{\partial X}+\frac{1}{2}\left(\frac{\partial W}{\partial X}\right)^{2}-Z \frac{\partial^{2} W}{\partial X^{2}},
$$

where $U$ is the displacement of the panel along the $X$ axis, and $W$ is the displacement of the panel along the $Z$-axis. Thus, the moment acting on the cross section of the panel (of unit width) may be expressed as

$$
M=\int_{-h / 2}^{h / 2} \sigma_{x x} Z d Z=-D \frac{\partial^{2} W}{\partial X^{2}} \text { (clockwise) }
$$

where $D$ is a coefficient characterizing the bending stiffness of the panel, $D=E h^{3} / 12\left(1-\nu^{2}\right)$.

Damping in this system has two sources: (a) external aerodynamic damping, which is due to the interaction between the panel and the flow, and (b) internal damping, which is due to the viscosity of material of the panel. The aerodynamic damping causes energy loss as a non-conservative force $F_{e}$ proportional to velocity of the panel. For certain flow velocities the aerodynamic damping may be negative, which leads to flutter. A more elaborate aerodynamic model has been used to account for the interaction between the panel and the flow. ${ }^{43,68}$ For simplicity, in the present analysis we consider that the flow is stationary, and the aerodynamic damping is simply proportional to the local velocity of the panel. Thus, the external damping is approximated as linear viscous damping of the form

$$
F_{e}=C_{e} \frac{\partial W}{\partial t}
$$

where $C_{e}$ is an external damping parameter. The material damping is considered to be a viscous damping 


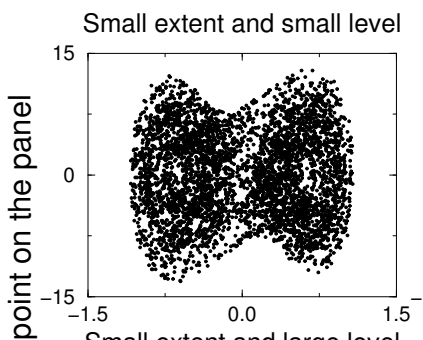

을 Small extent and large level

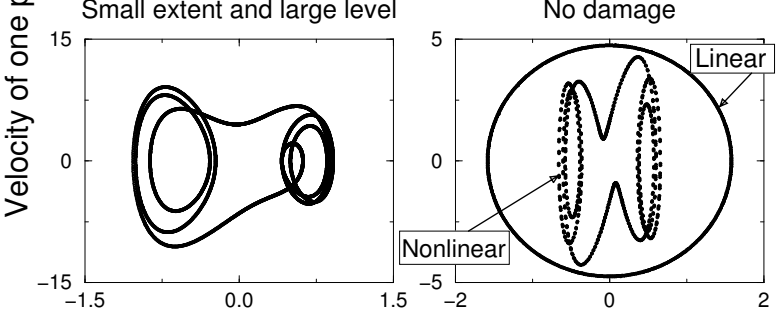

Displacement of one point on the panel / panel thickness

Fig. 4 The attractors of nonlinear systems are sensitive to damage presence, extent, and location, while linear models fail to model the dynamics

stress proportional to the strain rate, as follows

$$
\sigma_{x x}^{d}=\zeta \frac{\partial \epsilon_{x x}}{\partial t}
$$

where $\zeta$ is a material damping constant. Similar to Eq. 3, the moment $M^{d}$ corresponding to the damping stress and acting clockwise may be expressed as

$$
M^{d}=\int_{-h / 2}^{h / 2} \sigma_{x x}^{d} Z d Z=-C_{i} \frac{\partial^{3} W}{\partial X^{2} \partial t},
$$

where $C_{i}$ is defined as the internal damping parameter, and may be expressed as $C_{i}=\zeta h^{3} / 12$. Combining Eq. 3 and Eq. 4 , the total moment $\bar{M}$ acting clockwise on the cross section of the unit width panel is obtained as

$$
\bar{M}=M+M^{d}=-D \frac{\partial^{2} W}{\partial X^{2}}-C_{i} \frac{\partial^{3} W}{\partial X^{2} \partial t} .
$$

Fig. 5 shows the equilibrium of forces acting on an infinitesimal element of the panel (of unit width). This equilibrium of forces may be expressed as $\sum F_{Z}=0$, and may be used to obtain

$$
\frac{d T}{d X}=-C_{e} \dot{W}-\rho h \ddot{W}+P(X, t) .
$$

Also, the equilibrium of moments $\left(\sum M_{Y}=0\right)$ may be used to obtain

$$
N \frac{d W}{d X}-\frac{d \bar{M}}{d X}+T=0 .
$$

Differentiating Eq. 7 with respect to $X$ and substituting it and Eq. 5 into Eq. 6, the governing equation for the panel dynamics may be expressed as

$$
\begin{aligned}
& D W^{\prime \prime \prime \prime}+\rho h \ddot{W}+C_{i} \dot{W}^{\prime \prime \prime \prime}+ \\
& C_{e} \dot{W}-N W^{\prime \prime}-P(X, t)=0
\end{aligned}
$$

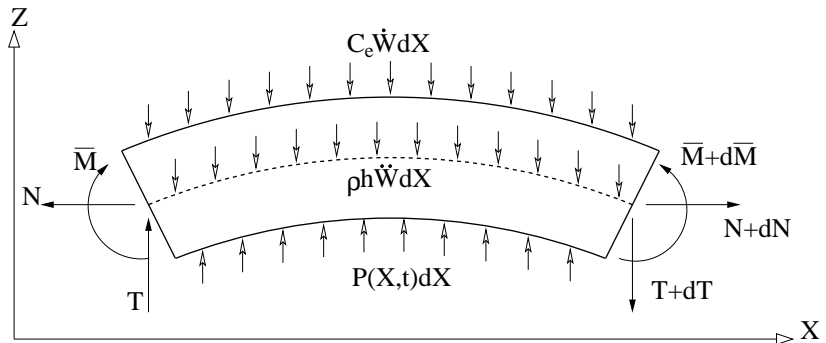

Fig. 5 Forces acting on an infinitesimal element of the panel including distributed transverse, axial, and bending forces

where $W^{\prime}$ and $\dot{W}$ represent the spatial and time derivatives of $W$, while $\rho$ is the mass density, and $P(X, t)$ is the transverse distributed loading. $N$ is the in-plane tension load, which is assumed to be uniform along the entire length of the panel. $N$ may be expressed as

$$
N=E h\left[\eta_{0}+\frac{1}{l} \int_{0}^{l} \frac{1}{2} W^{\prime 2}(\xi) d \xi\right],
$$

where the first term $\left(\eta_{0}\right)$ is the initial axial strain, and the second term is the stretching due to bending. Substituting Eq. 9 into Eq. 8, one obtains

$$
\begin{aligned}
D W^{\prime \prime \prime \prime} & +\rho h \ddot{W}+C_{i} \dot{W}^{\prime \prime \prime \prime}+C_{e} \dot{W}-E h \eta_{0} W^{\prime \prime} \\
& -\frac{E h}{2 l}\left[\int_{0}^{l} W^{2}(\xi) d \xi\right] W^{\prime \prime}-P(X, t)=0 .
\end{aligned}
$$

The boundary conditions corresponding to the pinnedpinned panel are

$$
\begin{array}{ll}
W(X=0, t)=0, & \bar{M}(X=0, t)=0, \\
W(X=l, t)=0, & \bar{M}(X=l, t)=0 .
\end{array}
$$

Next, Eq. 10 is nondimensionalized by introducing the nondimensional variables $x, w$, and $\tau$ defined as $x=X / l, w=W / h$, and $\tau=t / \sqrt{\rho h l^{4} / D}$. One obtains

$$
\begin{aligned}
w^{\prime \prime \prime \prime} & +\ddot{w}+C_{I} \dot{w}^{\prime \prime \prime \prime}+C_{E} \dot{w}-R_{x} w^{\prime \prime} \\
& -S\left[\int_{0}^{1} w^{\prime 2}(\xi) d \xi\right] w^{\prime \prime}-F(x, \tau)=0,
\end{aligned}
$$

where $w^{\prime}=\partial w / \partial x$, and $\dot{w}=\partial w / \partial \tau$. The nondimensional coefficients in Eq. 12 may be summarized as follows: internal damping $C_{I}=C_{i} / \sqrt{\rho h l^{4} D}$, external damping $C_{E}=C_{e} l^{2} / \sqrt{\rho h D}$, in-plane pre-load $R_{x}=$ $E h \eta_{0} l^{2} / D$, coefficient characterizing the stretching induced by bending $S=E h^{3} /(2 D)=6\left(1-\nu^{2}\right)$, and external excitation force $F(x, \tau)=l^{4} /(h D) P(x, \tau)$. The nondimensional boundary conditions may be expressed as

$$
\begin{aligned}
& w(x=0, \tau)=0, w^{\prime \prime}(x=0, \tau)+C_{I} \dot{w}^{\prime \prime}(x=0, \tau)=0 \\
& w(x=1, \tau)=0, w^{\prime \prime}(x=1, \tau)+C_{I} \dot{w}^{\prime \prime}(x=1, \tau)=0 .
\end{aligned}
$$



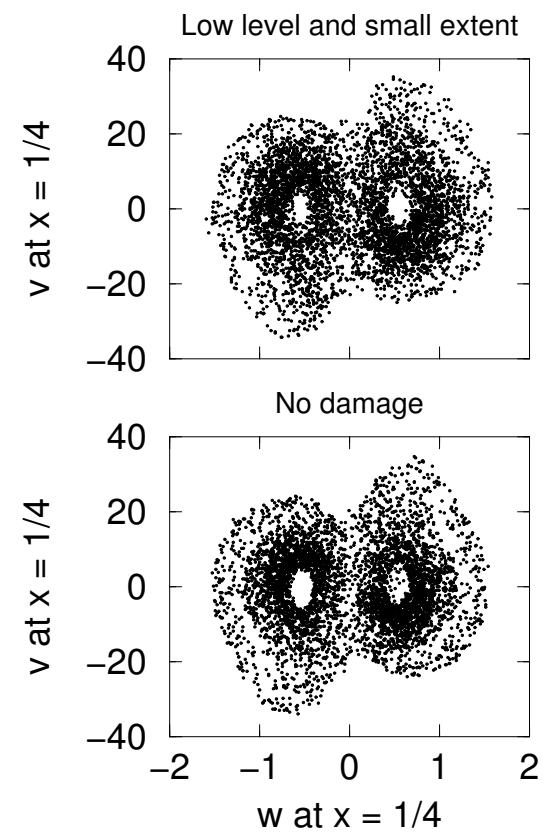

Low level and moderate extent

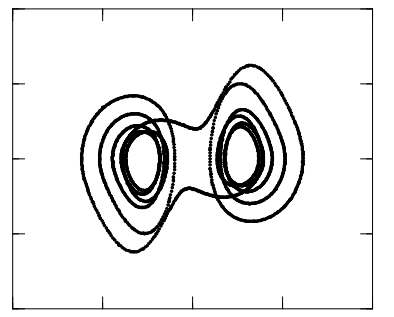

High level and moderate extent

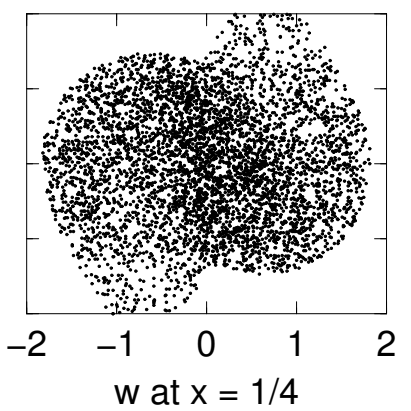

Low level and large extent

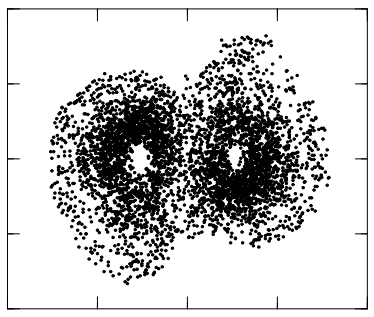

High level and large extent

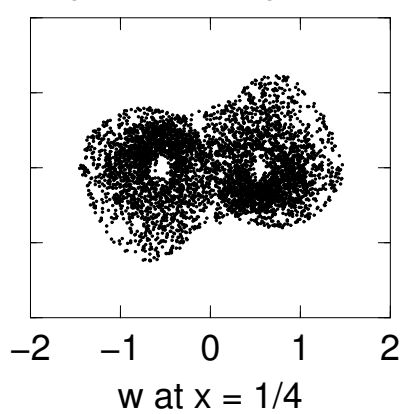

Fig. 6 Attractors sampled in time for a healthy panel and three damaged panels with low or high levels of damage and various extents of damage $(v=\dot{w})$

\section{Damage Model}

In many damage scenarios, yielding occurs in a small region of the panel, or a crack is initiated and propagates in the material. Such types of damage lead to a reduction of the local bending stiffness coefficient $\bar{D}$. The bending stiffness and Young's modulus for a damaged panel are denoted by $\bar{D}$ and $\bar{E}$. Similar to Eq. 10, the governing equation for the damaged region of the panel may be expressed as

$$
\begin{aligned}
\bar{D} W^{\prime \prime \prime \prime} & +\rho h \ddot{W}+C_{i} \dot{W}^{\prime \prime \prime \prime}+C_{e} \dot{W}-\bar{E} h \eta_{0} W^{\prime \prime} \\
& -\frac{\bar{E} h}{2 l}\left[\int_{0}^{l} W^{\prime 2}(\xi) d \xi\right] W^{\prime \prime}-P(X, t)=0 .
\end{aligned}
$$

The same nondimensional variables $x, w$, and $\tau$ defined for the undamaged panel are used to nondimensionalize Eq. 13. One obtains

$$
\begin{aligned}
S_{r} \cdot w^{\prime \prime \prime \prime} & +\ddot{w}+C_{I} \dot{w}^{\prime \prime \prime \prime}+C_{E} \dot{w}-S_{r} \cdot R_{x} w^{\prime \prime} \\
& -S_{r} \cdot S\left[\int_{0}^{1} w^{\prime 2}(\xi) d \xi\right] w^{\prime \prime}-F(x, \tau)=0
\end{aligned}
$$

where $S_{r}$ is referred to as a stiffness reduction factor, $S_{r}=\bar{D} / D$, and characterizes the damage level in the panel. No damage is present when $S_{r}$ equals unity, and small values of $S_{r}$ indicate large damage.

\section{Nonlinear Feedback Excitation}

Most current techniques for damage detection are based on observing the dynamics under ambient loads, or harmonic excitation. The main advantage of using a simple harmonic external excitation is that the excitation is easy to generate and measure. However, such an excitation is not best suited for interrogating a nonlinear system for the purpose of identifying small changes in its parameters. Furthermore, current vibration-based methods are designed for linear systems and minimize as much as possible the influence of nonlinearities in the dynamics during measurements. The method proposed herein is to exploit and enhance (where necessary) nonlinearities. The main advantage of using such an approach is a much enhanced sensitivity. To enhance nonlinearity, we propose an active approach where the system is actively interrogated by applying a nonlinear feedback excitation. As opposed to the usual external excitation, the nonlinear feedback excitation requires the active/online measurement of the the dynamics and a feedback loop. The specific form of the nonlinear feedback is dependent on each particular application. The excitation may include quadratic, cubic or other nonlinearities.

As opposed to the usual response to a harmonic excitation, the dynamics of the monitored system is chaotic during the interrogation phase. For linear or nonlinear systems undergoing limit cycle oscillations, the attractors are curves in the state space whose shape may be used as an indicator of damage. ${ }^{40}$ However, the sensitivity of the shape of these curves is limited ${ }^{68}$ because their shape is not very complex. Increasing the geometric complexity of the attractor of the dynamics is shown here to be a venue to increase sensitivity. Specifically, the nonlinear feedback excitation may be used to induce chaotic dynamics, which (usually) has attractors expanded spatially. ${ }^{69-71}$ The increased sensitivity to parametric variation is demonstrated in Fig. 7 where bifurcation diagrams are shown 

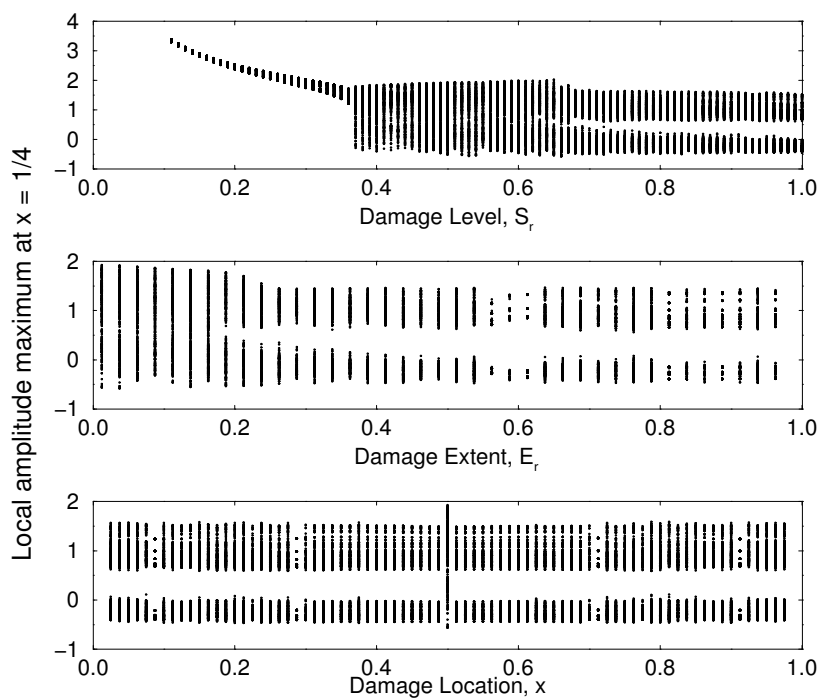

Fig. 7 Bifurcation diagram for varying damage level, extent and location, showing the dramatic changes in the dynamic attractor for varying damage characteristics

for varying damage level $S_{r}$, varying damage extent (i.e. the fraction of panel where loss of stiffness occurs - denoted by $E_{r}$ ), and varying damage location (along the panel). A clear distinction in the dynamics may be observed for various levels, extents and locations of damage. The changes revealed in the bifurcation diagrams are also reflected in the shape of the attractor of the dynamics.

The active interrogation of the system leads to changes in its dynamics so that the attractor (in state space or in embedded coordinates) is a complex geometric shape. This shape is used as a feature in a damage detection approach, i.e. given a (measured) attractor, damage is detected by comparing the shape of this attractor with its undamaged shape. This shape is complex, and its characterization is designed to provide the damage level as well as location. This approach is very different from other attractorbased methods such as chaotic excitation. Chaotic excitation has been used for damage detection with success, ${ }^{37,72-75}$ but the attractor variance, and Lyapunov exponents which were used as features could not predict the damage location, and their sensitivity was moderate. The proposed approach is radically different. For example, when a chaotic excitation is applied to a linear system, superposition still holds and each frequency component of the chaotic signal is filtered through the transfer function of the structure as any other type of external (non-feedback) excitation would be. However, in the nonlinear feedback approach, superposition does not hold. The excitation and the system are merged in a new system whose dynamics is very sensitive to changes in its parameters.

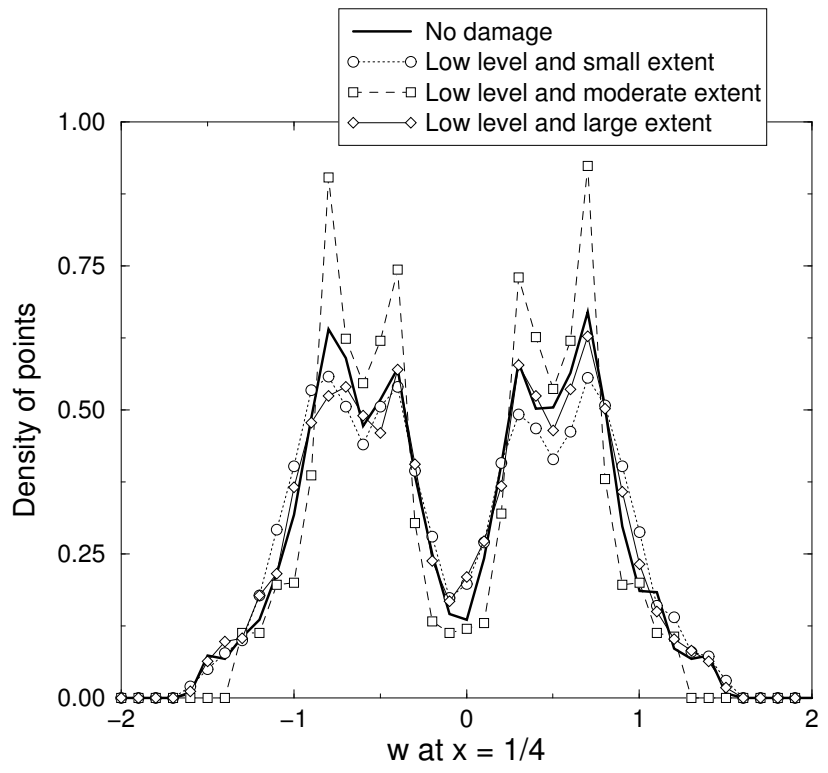

Fig. 8 Histogram of the point distribution in an attractor for the dynamics of a system with low damage and various extents

In order to make the dynamics of the panel sensitive to the level, the extent, and the location of the damage in the panel, a harmonic excitation with a nonlinear feedback force component is applied at the middle point of the panel. Thus, the transverse distributed loading $F(x, \tau)$ in Eq. 12 can be expressed as $F(\tau) \delta(x-1 / 2)$, where $F(\tau)$ is response function of the input force, and $\delta$ is the Dirac delta function.

The structural nonlinear term in Eq. 12, $S\left[\int_{0}^{1} w^{\prime 2}(\xi) d \xi\right] w^{\prime \prime}$, plays an important role in the complex dynamics of the panel. However, in cases such as a panel constrained by pinned-roller boundary conditions, the nonlinear effect is weak. When the structural nonlinearity and the in-plane pre-load are small, the panel dynamics subjected to a harmonic excitation force leads to harmonic oscillation. To enhance the sensitivity of these vibrations to parametric variations, a nonlinear feedback force is used in combination with the harmonic force. As a result, the input force may be expressed as

$$
\begin{aligned}
F(\tau)=F_{0} \sin \omega \tau & +\lambda w(1 / 2)^{2} w^{\prime \prime}(1 / 2) \\
& +\gamma w^{\prime \prime}(1 / 2)
\end{aligned}
$$

where $w(1 / 2)$ and $w^{\prime \prime}(1 / 2)$ are the displacement and the curvature at the middle point of the panel, and $\lambda$ and $\gamma$ are control parameters. The form of the nonlinear feedback force is drawn from the form of two terms in Eq. $12, S\left[\int_{0}^{1} w^{\prime 2}(\xi) d \xi\right] w^{\prime \prime}$ and $R_{x} w^{\prime \prime}$, corresponding to structural nonlinearity and axial pre-load. They are designed to increase the nonlinearity and enhance the instability in the linear panel. 


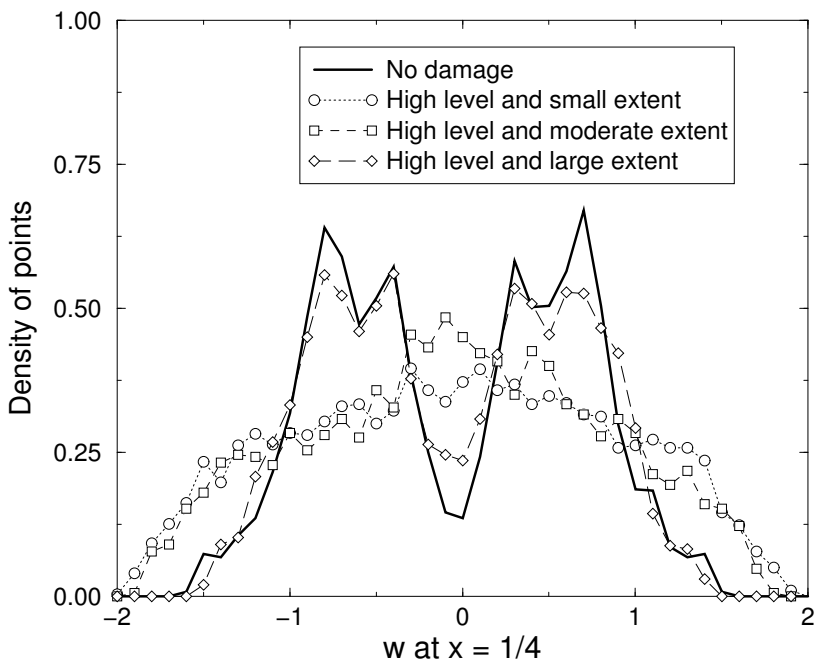

Fig. 9 Histogram of the point distribution in an attractor for the dynamics of a system with high damage and various extents

\section{Results}

To investigate the changes in the shape of attractors for various parametric values, we investigate first the local distribution of points in an attractor.

Fig. 6 shows the attractors of the dynamics sampled in time and obtained for a healthy panel and damaged panels with low and high levels of damage and various damage extents. The low level of damage is a $10 \%$ loss of stiffness (i.e. $S_{r}=0.9$ ), while the high level of damage is a $50 \%$ loss of stifness (i.e. $S_{r}=0.5$ ). Three values are considered for the extent of the damage: small extent ( $1.25 \%$ of panel length), moderate extent (5\% of panel length), and large extent ( $14 \%$ of panel length). The first key result demonstrated in Fig. 6 is that the attractor of the dynamics changes significantly when the extent of damage changes. Fig. 6 shows also the attractors obtained for panels with a high level of damage and various damage extents. The attractor undergoes geometric changes when parametric variations occur, such as is the case of the presence of damage. Also, Fig. 7 shows that the geometric changes in the attractor shape occur for all parameters of interest, i.e. the level, location, and extent of damage.

Histograms may be used to characterize the shape and distribution of points in an attractor. The histograms shown in Fig. 8 demonstrate that changes in attractors may be quantified for a (constant and low) level of damage and a varying extent of damage. The change in the attractor shape is dramatic when the damage varies from small to large extent. The moderate extent of damage affects the dynamics greatly, and leads to a collapse of the attractor onto a limit cycle, as shown in Fig. 6. More subtle changes occur for the case of high damage. Similar to the case of low damage, the attractor shape changes greatly when the

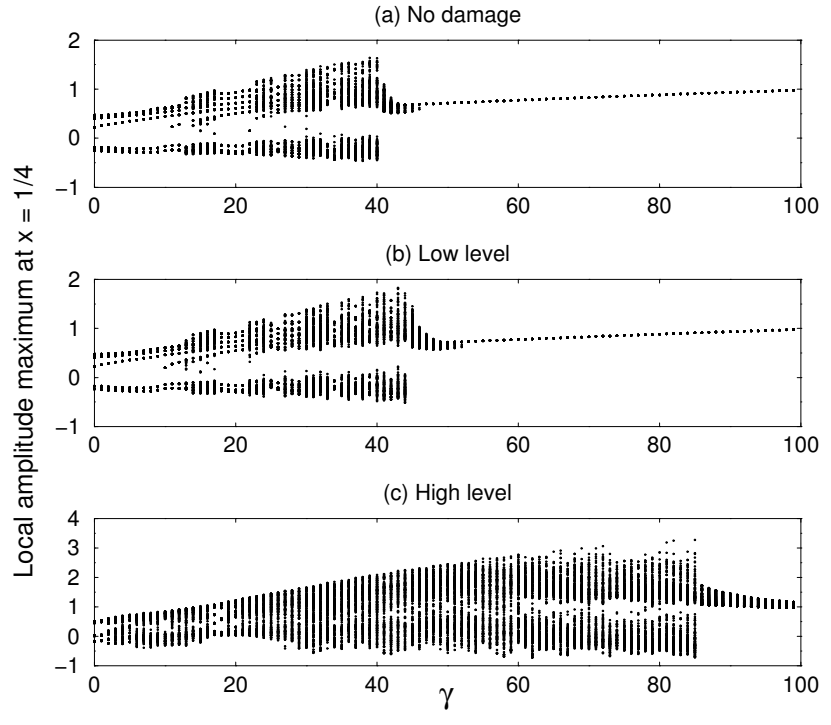

Fig. 10 Bifurcation diagram for varying characteristics of the nonlinear feedback excitation, which shows the dramatic changes in the attractor shape for three cases of damage

extent of damage is varied while the level of damage is constant and high, as shown in Fig. 9. Moreover, a comparison of the histograms shown in Fig. 8 and Fig. 9 reveals that the changes in the histograms are distinct for various damage levels when compared to various damage extents. Thus, both the level and the extent of damage may be detected.

Another approach to identify damage is to analyze bifurcation diagrams for varying characteristics of the nonlinear feedback excitation. Changing the characteristics of the excitation is much more easily done than changing system properties. Nevertheless, changing the excitation leads to a change in the dynamics of the global system (structure plus controller) as if a system parameter were changed because of the feedback nature of the excitation. Also, bifurcation diagrams reflect accurately qualitative and quantitative changes which may occur in the dynamics of nonlinear systems. ${ }^{44-46,76-80}$ In the context of damage detection, we construct bifurcation diagrams by varying the control parameters $\lambda$ and $\gamma$. Such diagrams are possible to measure because they require only the modification of the controller and not of the structure. To demonstrate this approach we focus on the detection of the onset limit cycle oscillations for varying parameters of the nonlinear feedback excitation. The local amplitude maxima at the quarter point location $(x=1 / 4)$ for various values of $\gamma$ and for three damage cases are shown in Fig. 10. The level of damage may be identified by observing that the onset of limit cycle oscillations occurs at clearly distinct values of $\gamma$ (of 45, 50 and over 100). 


\section{Conclusions}

A novel approach to actively interrogate a linear or nonlinear structure for the purpose of detecting parametric changes has been presented. The parametric changes identified are indicative of the presence, location and level of damage in the system. The main advantage of the proposed method is its high sensitivity to parametric variations, which makes it particularly well suited for detecting incipient damage.

Numerical examples have been presented for a panel with pinned-pinned end points forced by transverse loads and undergoing limit cycle oscillations and chaos. The nonlinear von Karman plate theory has been used to obtain a model for the panel, while damage has been modeled by a loss of stiffness in a portion of the plate. The sensitivity of this nonlinear system to parametric changes (enhanced by nonlinear feedback excitation) has been shown to be an effective tool in detecting structural changes and identifying stiffness loss in a thermo-shielding panel.

Most of the current studies of such problems are based on linear theories and linear structures. In contrast, the results presented are obtained using nonlinear and chaotic dynamics, and have the advantage of an increased accuracy in detecting damage and monitoring structural health. The sensitivity obtained by enhancing nonlinear dynamics and exploiting the features of chaotic dynamics is much higher than the sensitivity of linear analyses.

\section{References}

${ }^{1}$ Farrar, C. R., Doebling, S. W., and Nix, D. A., "VibrationBased Structural Damage Identification," Philosophical Transactions of the Royal Society of London: A - Mathematical, Physical and Engineering Sciences, Vol. 359, No. 1778, 2001, pp. 131-149.

${ }^{2}$ Zimmerman, D. C., "Looking into the Crystal Ball: The Continued Need for Multiple Viewpoints in Damage Detection," Key Engineering Materials: Damage Assessment of Structures, Vol. 167-168, No. 1, 1999, pp. 76-90.

${ }^{3}$ Doebling, S. W., Farrar, C. R., Prime, M. B., and Shevitz, D. W., "Damage Identification and Health Monitoring of Structural and Mechanical Systems from Changes in Their Vibration Characteristics: A Literature Review," Report LA-13070-MS, Los Alamos National Laboratories, Los Alamos, NM, 1996.

${ }^{4}$ Roemer, M. J., Kacprzynski, G. J., Schoeller, M., Howe, R., and Friend, R., "Advanced Test Cell Diagnostics for Gas Turbine Engines (USAF Automated Jet Engine Test Strategy AJETS," Proceedings of IGTI 2001 Turbo Expo, Vol. 1, ASME, New Orleans, LA, 2001, pp. 1-10.

${ }^{5}$ Kacprzynski, G. J., Roemer, M. J., Hess, A. J., and Bladen, K. R., "Extending FMECA - Health Management Design Optimization for Aerospace Applications," Proceedings of IEEE Aerospace Conference, Vol. 1, IEEE, Big Sky, MO, 2001, pp. $1-8$.

${ }^{6}$ Loh, C. H. and Tou, I. C., "A System Identification Approach to the Detection of Changes in both Linear and Nonlinear Structural Parameters," Earthquake Engineering and Structural Dynamics, Vol. 24, No. 1, 1995, pp. 85-97.

${ }^{7}$ Pandey, A. K. and Biswas, M., "Damage Detection in Structures Using Changes in Flexibility," Journal of Sound and Vibration, Vol. 169, No. 1, 1994, pp. 3-17.
${ }^{8}$ Smyth, A. W., Masri, S. F., Caughey, T. K., and Hunter, N. F., "Surveillance of Mechanical Systems on the Basis of Vibration Signature Analysis," Journal of Applied Mechanics, Vol. 67 , No. 3, 2000, pp. 540-551.

${ }^{9}$ Amaravadi, V. K., Mitchell, K., Rao, V. S., and Derriso, M. M., "Structural Integrity Monitoring of Composite Patch Repairs Using Wavelet Analysis and Neural Networks," Proceedings of SPIE: Smart Structures and Materials 2002: Smart Structures and Integrated Systems, edited by L. P. Davies, Vol. 4701, San Diego, California, 2002, pp. 156-166.

${ }^{10}$ Amaravadi, V. K., Rao, V. S., Koval, L. R., and Derriso, M. M., "Structural Health Monitoring Using Wavelet Transforms," Proceedings of SPIE: Smart Structures and Materials 2001: Smart Structures and Integrated Systems, edited by L. P. Davis, Vol. 4327, Newport Beach, California, 2001, pp. 258-269.

${ }^{11}$ Amizic, B., Amaravadi, V. K., Rao, V. S., and Derriso, M. M., "Two-Dimensional Wavelet Mapping Techniques for Damage Detection in Structural Systems," Proceedings of SPIE: Smart Structures and Materials 2002: Modeling, Signal Processing, and Control, edited by V. S. Rao, Vol. 4693, San Diego, California, 2002, pp. 267-278.

${ }^{12}$ Sohn, H. and Farrar, C. R., "Damage Diagnosis Using Time Series Analysis of Vibration Signals," Smart Materials and Structures, Vol. 10, No. 3, 2001, pp. 446-451.

${ }^{13}$ Cao, T. T. and Zimmerman, D. C., "Procedure to Extract Ritz Vectors from Dynamic Testing Data," Journal of Structural Engineering, Vol. 125, No. 12, 1999, pp. 1393-1400.

${ }^{14}$ Sadeghi, M. H. and Fassois, S. D., "Geometric Approach to Nondestructive Identification of Faults in Stochastic Structural Systems," AIAA Journal, Vol. 35, No. 4, 1997, pp. 700-705.

${ }^{15}$ Tanaka, S. and Muller, P. C., "Fault Detection in Linear Discrete Dynamic Systems by a Reduced Order Generalized Likelihood Ratio Method," International Journal of Systems Science, Vol. 24, No. 4, 1993, pp. 721-732.

${ }^{16}$ Abdalla, M. O., Grigoriadis, K. M., and Zimmerman, D. C., "Enhanced Structural Damage Detection Using Alternating Projection Methods," AIAA Journal, Vol. 36, No. 7, 1998, pp. 1305-1311.

${ }^{17}$ Ghanem, R. and Shinozuka, M., "Structural System Identification: Theory," ASCE Journal of Engineering Mechanics, Vol. 121, No. 2, 1995, pp. 255-264.

${ }^{18}$ Kim, H. M., Bartkowicz, T. J., Smith, S. W., and Zimmerman, D. C., "Health Monitoring of Large Structures," Journal of Sound and Vibration, Vol. 29, No. 4, 1995, pp. 18-21.

${ }^{19}$ van Overschee, P. and DeMoor, B., Subspace Identification for Linear Systems: Theory, Implementation and Applications, Kluwer, New York, 1996.

${ }^{20}$ Pappa, R. P., James, G. H., and Zimmerman, D. C., "Autonomous Modal Identification of the Space Shuttle Tail Rudder," Journal of Spacecraft and Rockets, Vol. 35, No. 2, 1998, pp. $163-169$.

${ }^{21}$ Patton, R. J. and Chen, J., "Robust Fault-Detection of Jet Engine Sensor Systems Using Eigenstructure Assignment," Journal of Guidance Control and Dynamics, Vol. 15, No. 6, 1992, pp. 1491-1497.

${ }^{22}$ Zimmerman, D. C., "Model Validation and Verification of Large and Complex Space Structures," Inverse Problems in Engineering, Vol. 8, No. 2, 2000, pp. 93-118.

${ }^{23}$ Zimmerman, D. C. and Simmermacher, T., "Model Correlation Using Multiple Static Load and Vibration Tests," AIAA Journal, Vol. 33, No. 11, 1995, pp. 2182-2188.

${ }^{24}$ Zimmerman, D. C., Yap, K., and Hasselman, T., "Evolutionary Approach for Model Refinement," Mechanical Systems and Signal Processing, Vol. 13, No. 4, 1999, pp. 609-625.

${ }^{25}$ Agbabian, M. S., Masri, S. F., Miller, R. F., and Caughey, T. K., "System Identification Approach to Detection of Structural Changes," ASCE Journal of Engineering Mechanics, Vol. 117, No. 2, 1990, pp. 370-390.

${ }^{26}$ Feeny, B. F., Yuan, C. M., and Cusumano, J. P., "Parametric Identification of an Experimental Magneto-Elastic Oscil- 
lator," Journal of Sound and Vibration, Vol. 247, No. 5, 2001, pp. 785-806.

${ }^{27}$ Ljung, L., System Identification - Theory for the User, Prentice Hall, New York, 1999.

${ }^{28}$ Masri, S. F. and Caughey, T. K., "A Non-Parametric Identification Technique for Nonlinear Dynamic Problems," Journal of Applied Mechanics, Vol. 46, No. 2, 1979, pp. 433-447.

${ }^{29}$ Masri, S. F., Bekey, G. A., Sassi, H., and Caughey, T. K., "Non-Parametric Identification of a Class of Nonlinear Multidegree Dynamic Systems," Earthquake Engineering and Structural Dynamics, Vol. 10, No. 1, 1982, pp. 1-30.

${ }^{30}$ Masri, S. F., Miller, R. K., Saud, A. F., and Caughey, T. K., "Identification of Nonlinear Vibrating Structures: Part I: Formulation," Journal of Applied Mechanics, Vol. 109, No. 54, 1987, pp. 918-922.

${ }^{31}$ Smyth, A. W., Masri, S. F., Chassiakos, A. G., and Caughey, T. K., "On-line Parametric Identification of MDOF Nonlinear Hysteretic Systems," ASCE Journal of Engineering Mechanics, Vol. 125, No. 2, 1999, pp. 133-142.

${ }^{32}$ Atalla, M. J. and Inman, D. J., "On Model Updating Using Neural Networks," Mechanical Systems and Signal Processing, Vol. 12, No. 1, 1998, pp. 135-161.

${ }^{33}$ Marwala, T. and Hunt, H. E. M., "Fault Identification Using Finite Element Models and Neural Networks," Mechanical Systems and Signal Processing, Vol. 13, No. 3, 1999, pp. 475490 .

${ }^{34}$ Zang, C. and Imregun, M., "Structural Damage Detection Using Artificial Neural Networks and Measured FRF Data Reduced via Principal Component Projection," Journal of Sound and Vibration, Vol. 242, No. 5, 2001, pp. 813-827.

${ }^{35}$ Chancellor, R. S., Alexander, R. M., and Noah, S. T., "Detecting Parameter Changes Using Experimental Nonlinear Dynamics and Chaos," Journal of Vibration and Acoustics, Vol. 118, No. 3, 1996, pp. 375-383.

${ }^{36}$ Pecora, L. M. and Caroll, T. L., "Discontinuous and Nondifferentiable Functions and Dimension Increase Induced by Filtering Chaotic Data," Chaos, Vol. 6, No. 3, 1996, pp. 432-439.

${ }^{37}$ Trickey, S. T., Todd, M., Seaver, M., and Nichols, J., "Geometric Time Domain Methods of Vibration Based Damage Detection," Proceedings of the SPIE 9-th Smart Structures and Materials Conference, Vol. 1, San Diego, California, 2002, pp. $1-9$.

${ }^{38}$ Wang, W. J., Wu, Z. T., and Chen, J., "Fault Identification in Rotating Machinery Using the Correlation Dimension and Bispectra," Nonlinear Dynamics, Vol. 25, No. 4, 2001, pp. 383393.

${ }^{39}$ Rizos, P. F., Aspragathos, N., and Dimarogonas, A. D., "Identification of Crack Location and Magnitude in a Cantilever Beam from the Vibration Modes," Journal of Sound and Vibration, Vol. 138, No. 3, 1990, pp. 381-388.

${ }^{40}$ Kesaraju, R. V. and Noah, S. T., "Characterization and Detection of Parameter Variations of Nonlinear Mechanical Systems," Nonlinear Dynamics, Vol. 6, No. 4, 1994, pp. 433-457.

${ }^{41}$ van de Vors, T. E. L., Heertjes, M. F., van Campen, D. H., Kraker, A., and Fey, R. H. B., "Experimental and Numerical Analysis of the Steady State Behaviour of a Beam System With Impact," Journal of Sound and Vibration, Vol. 212, No. 2, 1998, pp. $321-336$.

${ }^{42}$ van de Vors, T. E. L., van Campen, D. H., and de Kraker, A., "Periodic Solutions of a Multi-DOF Beam System With Impact," Journal of Sound and Vibration, Vol. 192, No. 5, 1996, pp. $913-926$.

${ }^{43}$ Epureanu, B. I., Tang, L. S., and Paidoussis, M. P., "Coherent Structures and Their Influence on the Dynamics of Aeroelastic Panels," International Journal of Non-Linear Mechanics, 2004, to appear.

${ }^{44}$ Guckenheimer, J. and Holmes, P., Nonlinear Oscillations, Dynamical Systems, and Bifurcations of Vector Fields, Springer-Verlag, New York, 1983.
${ }^{45}$ Strogatz, S. H., Nonlinear Dynamics and Chaos, AddisonWesley, Reading, MA, 1994.

${ }^{46}$ Wiggins, S., Introduction to Applied Nonlinear Dynamical Systems and Chaos, Springer-Verlag, New York, 1990.

${ }^{47}$ Dowell, E. H., A Modern Course in Aeroelasticity, Kluwer Academic Publishers, Dordrecht, ii ed., 1989.

${ }^{48}$ Dowell, E. H., Aeroelasticity of Plates and Shells, Noordhoff International Publishing, Leyden, 1975.

${ }^{49}$ Dowell, E. H., "Flutter of a Buckled Plate as an Example of Chaotic Motion of a Deterministic Autonomous System," Journal of Sound and Vibration, Vol. 85, No. 3, 1982, pp. 333-344.

${ }^{50} \mathrm{Kim}, \mathrm{D} . \mathrm{H}$. and Lee, I., "Transonic and Low-Supersonic Aeroelastic Analysis of a Two-Degree-of-Freedom Airfoil With a Freeplay Non-Linearity," Journal of Sound and Vibration, Vol. 234, No. 5, 2000, pp. 859-880.

${ }^{51}$ Silva, W. A., "Reduced-Order Models Based on Linear and Nonlinear Aerodynamic Impulse Responses," Proceedings of the CEAS/AIAA/ICASE/NASA Langley International Forum on Aeroelasticity and Structural Dynamics, AIAA, Copenhagen, Denmark, 1999, pp. 1731-1738.

${ }^{52}$ Bolotin, V. V., Grishko, A. A., Kounadis, A. N., and Gantes, C. J., "The Fluttering Panel as a Continuous Nonlinear Nonconservative System," Journal of Vibration and Control, Vol. 7, No. 2, 2001, pp. 233-247.

${ }^{53}$ Bolotin, V. V., Grishko, A. A., Kounadis, A. N., and Gantes, C. J., "Non-linear Panel Flutter in Remote PostCritical Domains," International Journal of Non-linear Mechanics, Vol. 33, No. 5, 1998, pp. 753-764.

${ }^{54}$ Bolotin, V. V., Grishko, A. A., Kounadis, A. N., and Gantes, C. J., "Influence of Initial Conditions on the Postcritical Behavior of a Nonlinear Aeroelastic System," Nonlinear Dynamics, Vol. 15, No. 1, 1998, pp. 63-81.

${ }^{55}$ Epureanu, B. I., Tang, L. S., and Paidoussis, M. P., "Observations of the Dynamics of Panels in Supersonic Flow," Proceedings of the Second M.I.T. Conference on Computational Fluid and Solid Mechanics, Vol. 2, Cambridge, Massachusetts, 2003, pp. $1332-1336$.

${ }^{56}$ Niho, T., Horie, T., and Tanaka, Y., "Numerical Instability of Magnetic Damping Problem of Elastic Plate," IEEE Transactions on Magnetics, Vol. 36, No. 4, pt. 1, 2000, pp. 1374-1377.

${ }^{57}$ Reynolds, R. R., Virgin, L. N., and Dowell, E. H., "Highdimensional Chaos Can Lead to Weak Turbulence," Nonlinear Dynamics, Vol. 4, No. 1, 1993, pp. 531-546.

${ }^{58}$ Reynolds, R. R. and Dowell, E. H., "Nonlinear Aeroelastic Response of Panels," Proceedings of 34-th AIAA/ASME/ $A S C E / A S C$ Structures, Structural Dynamics, and Materials Conference, Vol. 1, AIAA, La Jolla, CA, 1993, pp. 1-13.

${ }^{59} \mathrm{Jang}, \mathrm{M}$. J. and Chen, C. K., "Bifurcation Analysis in Flexible Rotor Supported by Active Magnetic Bearing," International Journal of Bifurcation and Chaos, Vol. 11, No. 8, 2001, pp. 2163-2178.

${ }^{60}$ Paidoussis, M. P., Fluid-Structure Interactions: Slender Structures and Axial Flow, Academic Press, San Diego, CA, 1998.

${ }^{61}$ Paidoussis, M. P. and Botez, R. M., "Chaotic Dynamics of Articulated Cylinders in Confines Axial Flow," Journal of Fluids and Structures, Vol. 7, 1993, pp. 719-750.

${ }^{62}$ Paidoussis, M. P., "Stability of a Chain of Cylinders Travelling Underwater," Proceedings of 5-th International Offshore Mechanics and Arctic Engineering Symposium, Vol. 1, ASME, New Orleans, LA, 1986, pp. 483-490.

${ }^{63}$ Copeland, G. S. and Moon, F. C., "Chaotic Flow-Induced Vibration of a Flexible Tube With End Mass," Journal of Fluids and Structures, Vol. 6, No. 3, 1992, pp. 705-718.

${ }^{64}$ Langthjem, M. A. and Sugiyama, Y., "Dynamic Stability of Columns Subjected to Follower Loads: A Survey," Journal of Sound and Vibration, Vol. 238, No. 5, 2000, pp. 809-851.

${ }^{65}$ Thothandri, M. and Moon, F. C., "An Investigation of Nonlinear Models for a Cylinder Row in a Cross Flow," Journal 
of Pressure Vessel Technology, Vol. 121, No. 1, 1999, pp. 133141.

${ }^{66}$ Turner, K. L., Miller, S. A., Hartwell, P. G., MacDonald, N. C., Strogatz, S. H., and Adams, S. G., "Five Parametric Resonances in a Microelectromechanical System," Nature, Vol. 396, No. 6707, 1998, pp. 149-152.

${ }^{67}$ Miller, S. A., Turner, K. L., and MacDonald, N. C., "Microelectromechanical Scanning Probe Instruments for Array Architectures," Review of Scientific Instruments, Vol. 68, No. 11, 1997, pp. 4155-4162.

${ }^{68}$ Epureanu, B. I., Tang, L. S., and Paidoussis, M. P., "Exploiting Chaotic Dynamics for Detecting Parametric Variations in Aeroelastic Systems," AIAA Journal, 2004, to appear.

${ }^{69}$ Epureanu, B. I. and Dowell, E. H., "System Identification for Ott-Grebogi-Yorke Controller Design," Physical Review E, Vol. 56, No. 5, 1997, pp. 5327-5331.

${ }^{70}$ Epureanu, B. I. and Dowell, E. H., "On the Optimality of the OGY Control Scheme," Physica D, Vol. 116, No. 1-2, 1998, pp. 1-7.

${ }^{71}$ Epureanu, B. I. and Dowell, E. H., "Optimal MultiDimensional OGY Controller for Stabilizing Fully Unstable Limit Cycles," Physica D, Vol. 139, No. 1-2, 2000, pp. 87-96.

${ }^{72} \operatorname{Logan}$, D. and Mathew, J., "Using the Correlation Dimension for Vibration Fault Diagnosis of Rolling Element Bearings: Basic Concepts," Mechanical Systems and Signal Processing, Vol. 10, No. 3, 1996, pp. 241-250.

${ }^{73}$ Schouten, J. C. and van den Bleek, C. M., "Monitoring the Quality of Fluidization Using the Short-Term Predictability of Pressure Fluctuations," AICHE Journal, Vol. 44, No. 1, 1998, pp. $48-60$.

${ }^{74}$ van Ommen, J. R., Coppens, M. O., van den Bleek, C. M., and Schouten, J. C., "Early Warning of Agglomeration in Fluidized Beds by Attractor Comparison," AICHE Journal, Vol. 46, No. 11, 2000, pp. 2183-2197.

${ }^{75}$ Yang, B., Suh, C. S., and Chan, A. K., "Characterization and Detection of Crack-Induced Rotary Instability," Journal of Vibration and Acoustics, Vol. 124, No. 1, 2002, pp. 40-48.

${ }^{76}$ Berge, P., Pomeau, Y., and Vidal, C., Order Within Chaos, John Wiley \& Sons, New York, 1984.

${ }^{77}$ Moon, F. C., Chaotic and Fractal Dynamics: An Introduction for Applied Scientists and Engineers, John Wiley \& Sons, New York, 1992.

${ }^{78}$ Nayfeh, A. H., Nonlinear Interactions, John Wiley \& Sons, New York, 2000.

${ }^{79}$ Ott, E., Chaos in Dynamical Systems, Cambridge University Press, New York, 1993.

${ }^{80}$ Thompson, J. M. T. and Stewart, H. B., Nonlinear Dynamics and Chaos, John Wiley \& Sons, New York, 1986. 